\title{
Enumerative Encoding of TMTR Codes for Optical Recording Channel
}

\author{
Hui-Feng Tsai \\ Department of Computer Science and Information Engineering, Ching Yun University, Jhongli City 32097, Taiwan \\ Correspondence should be addressed to Hui-Feng Tsai, hftsai@cyu.edu.tw \\ Received 26 July 2010; Accepted 19 September 2010 \\ Academic Editor: Magnus Jansson \\ Copyright () 2010 Hui-Feng Tsai. This is an open access article distributed under the Creative Commons Attribution License, \\ which permits unrestricted use, distribution, and reproduction in any medium, provided the original work is properly cited. \\ We propose a new time-varying maximum transition run (TMTR) code for DVD recording systems, which has a rate 8/11 higher \\ than the EFMPlus code and a lower power spectral density (PSD) at low frequencies. An enumeration method for constructing \\ the new TMTR code is presented. Computer simulations indicate that the proposed TMTR code outperforms the EFMPlus code \\ in error performance when applied to partial response optical recording channels.
}

\section{Introduction}

In data storage systems, a modulation code is known as $(d, k)$-constrained code, where $d$ and $k$ represent the maximal and minimal number of zeros between two consecutive ones. The main function of a $(d, k)$ modulation code is to improve the recording density and increase the storage capacity. The timing information could also be controlled using a $(d, k)$ modulation code. For example, magnetic tape and disk systems often adopt $(1,7)$ or $(2,7)$ codes, while optical systems such as CD and DVD usually employ $(2,10)$ EFM (Eight-to-Fourteen Modulation) or $(2,10)$ EFMPlus [1] modulation codes.

Recent research on the $(d, k)$ modulation code has focused on the time-varying maximum transition run (TMTR) code [2-9], which can be treated as a $(0, k)$ modulation code. The TMTR code matched to the partial response channel can delete some dominant error events and enhance the Euclidian distance of the partial response channel to the matched filter bound. As a result, a coding gain over the conventional scheme can be obtained when the time-varying Viterbi detector is applied to the TMTRcoded partial response channel. In a previous work [10], we proposed a new time-varying maximum transition run (TMTR) code with $\left(k_{1}^{\text {even }}=1, k_{1}^{\text {odd }}=2, k\right)$ constraint for DVD recording systems, which has rate $8 / 11$ higher than the EFMPlus code and a lower power spectral density (PSD) at low frequencies. The $\left(k_{1}^{\text {even }}=1, k_{1}^{\text {odd }}=2, k\right)$ TMTR code was realized with a look-up table, and the $k$ constraint was not considered during construction. In this paper, instead of a look-up table we present an enumeration method for constructing the $\left(k_{1}^{\text {even }}=1, k_{1}^{\text {odd }}=2, k\right)$ codes. Based on this construction, a rate $8 / 11$ code with $k=7$ is found. The proposed code can achieve better timing recovery performance. We show that 387 surviving words exist with length 11 from the construction technique. This new method needs one bit of memory for encoding, but no memory is required for decoding. An enumerating algorithm is used for encoding/decoding, and a look-up table is not required.

The rest of this paper is organized as follows. In Section 2, we briefly describe the $\left(k_{1}^{\text {even }}=1, k_{1}^{\text {odd }}=2, k\right)$ TMTR codes for partial response (PR) optical recording channels. In Section 3, an outline of the design methodology for constructing a high-rate TMTR code is presented. We illustrate concatenation problem between codewords and provide a solution. Section 4 introduces an enumerative coding method for TMTR codes. In Section 5, the power spectral density (PSD) of the rate $8 / 11$ code is evaluated and compared with the EFMPlus code. An error performance comparison between uncoded, TMTR-coded, and EFMPluscoded EPRII optical recording channel is presented in Section 6. The conclusion is provided in Section 7. 


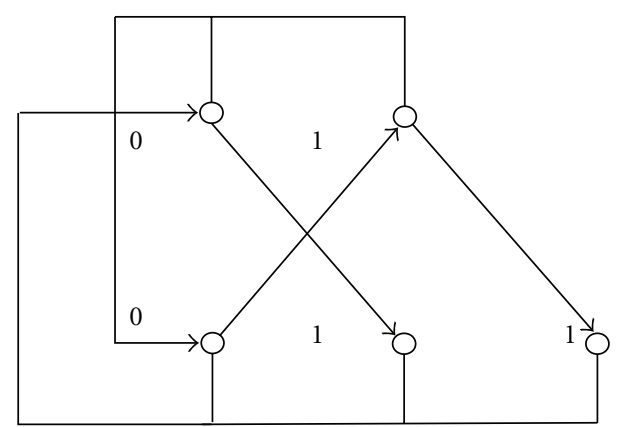

FIGURE 1: FSTD of TMTR constraint.

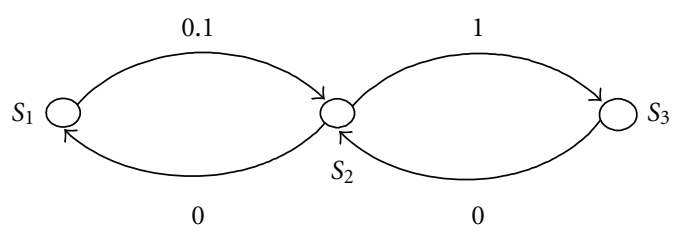

Figure 2: Simplified FSTD with TMTR constraint.

\section{TMTR Codes for Partial Response Optical Recording Channels}

The maximum transition run (MTR) method is a coding method, which limits the number of consecutive potential variations being not greater than $k$. The time-varying maximum transition run (TMTR) is a further modification of the MTR, which sets different constraints for the number of consecutive variations depending upon whether it starts at an odd or even position. For example, $\left(k_{1}^{\text {even }}, k_{1}^{\text {odd }}\right)$ TMTR constraints mean that the number of consecutive 1s starting at an even position is not greater than $k_{1}^{\text {even }}$ and the number of consecutive $1 \mathrm{~s}$ starting at an odd position is not greater than $k_{1}^{\text {odd }}$. The method can increase the minimum distance of the encoded system to an upper matched filter bound (MFB); therefore, it has the distance enhancing property. The TMTR constraint can be described using a finite state transition diagram (FSTD), given in Figure 1. The vertices at the top of the diagram represent even positions, and the number of 1 s starting at the even positions can be 1 only, satisfying the constraint of $k_{1}^{\text {even }}=1$. The vertex at the bottom of the diagram represents odd positions, and the number of 1 s starting at the odd positions can be 1 or 2 , satisfying the constraint of $k_{1}^{\text {odd }}=2$. Figure 2 shows a simplified FSTD with the $\left(k_{1}^{\text {even }}=1, k_{1}^{\text {odd }}=2\right)$ TMTR constraints.

Cideciyan et al. [11] suggested an advanced signal processing technique, the partial response and maximal likelihood (PRML) channel, to further increase the recording densities and reliability over that achieved by the conventional peak detector. The signal processing technique employing the PRML channel has become a standard widely used in most of today's data storage systems. The most popular partial response system for optical recording has the form $(1+D)^{n}$, where $n$ is a nonnegative integer. The PR systems with $n=$ 2 and 3 are referred to as the PRII and EPRII systems, respectively. Karabed and Siegel [12] proposed a class of modulation codes that take advantage of the well-defined spectral nulls presented in partial response channels. The time-varying maximum transition run (TMTR) code [2-9], which can be treated as a $(0, k)$ modulation code, has recently been studied for partial response channels. The TMTR code matched to the partial response channel can delete some dominant error events and enhance the Euclidian distance of the partial response channel.

Vannucci and Foschini [13] described a powerful algorithm to search for the minimum Euclidean distance $d_{\mathrm{min}}^{2}$ for $(1+D)^{n}$ partial response channels. They found that the shortest error event achieving $d_{\text {min }}^{2}$ has the type of " $\ldots 0+$ $-0 \ldots$ " for most of $(1+D)^{n}$ partial response channels. As a matter of fact they found that those error events of the form " $\ldots 0+-(+-) 0 \ldots$ " always have a distance less than the matched filter bound $d_{\mathrm{MFB}}^{2}$ which is defined as the distance corresponding to the one-bit error event.

If the error event " $\ldots 0+-0 \ldots$ " can be forbidden to occur in coded sequences for $(1+D)^{n}$ partial response channels, the minimum distance of the channels can be increased to $d_{\mathrm{MFB}}^{2}$ resulting in a coding gain of $10 \log \left(d_{\mathrm{MFB}}^{2} / d_{\mathrm{min}}^{2}\right) \mathrm{dB}$. With NRZI modulation there are four pairs of binary coded sequences, which could generate the error event " $\ldots 0+$ $-0 \ldots$, , shown as follows:

$\begin{array}{llll}110 & 111 & 010 & 011 \\ 011 & 010 & 111 & 110\end{array}$

The TMTR modulation code with constraint $\left(0, k_{1}^{\text {even }}=\right.$ $1, k_{1}^{\text {odd }}=2$ ) can be used to forbid the occurrence of sequences 111 and 011 , and as a result error event " ...0 + - $0 \ldots$ " would not occur in the detection of $(1+D)^{3}$ channels, and a coding gain of $3 \mathrm{~dB}$ can be obtained. The channel capacity of the $\left(0, k_{1}^{\text {even }}=1, k_{1}^{\text {odd }}=2\right)$ TMTR code is equal to 0.7929 , which indicates that a codeword with length 11 bits at least is required to encode or represent a byte (8-bit) message.

\section{Construction for $\left(k_{1}^{\text {even }}=1, k_{1}^{\text {odd }}=2, k\right)$ TMTR Codes}

A TMTR code is specified as $\left(k_{1}^{\text {even }}=1, k_{1}^{\text {odd }}=2, k\right)$ constraint, where $k$ is the maximum number of consecutive zeros, $k_{1}^{\text {even }}$ and $k_{1}^{\text {odd }}$ constraints represent the maximum numbers of consecutive ones starting from an even position and an odd position, respectively. This construction is based upon $\left(k_{1}^{\text {even }}=1, k_{1}^{\text {odd }}=2, k ; r_{1}, r_{0}, l_{1}, l_{0}\right)$ constraint. Both $r_{1}$ and $r_{0}$ represent the maximum number of ones after the last zero and the maximum number of zeros after the last one, respectively. In similar, parameters $l_{1}$ and $l_{0}$ represent the maximum number of ones before the first zero and the maximum number of zeros before the first one. Any two sequences satisfying $\left(k_{1}^{\text {even }}=1, k_{1}^{\text {odd }}=2, k ; r_{1}, r_{0}, l_{1}, l_{0}\right)=$ $(1,2, k ; 1, k-1,1, k)$ constraint can be freely concatenated without violating the $\left(k_{1}^{\text {even }}=1, k_{1}^{\text {odd }}=2, k\right)$ constraint. In order to reduce the consecutive zero length in the sequences after concatenation, the following substitution rule is applied: assume that a sequence $\mathbf{y}$ is followed by 
a sequence $\mathbf{x}$, then

(1) if $\mathbf{x}$ has more than 2 zeros before the first one, and the last bit of $\mathbf{y}$ is a zero, then flip the first 2 bits of $\mathbf{x}$ into two ones, for example, $(000 \ldots 101) \rightarrow(110 \ldots 101)$;

(2) otherwise, use $\mathbf{x}$ as the encoder output.

In the case of (1), a long sequence of consecutive zeros is spread into two parts by " 11 " in the beginning of the 2 nd sequence. Because $l_{1}=1$, a sequence beginning with " 11 " is not an original code.

Rates of some constructed codes using this method are listed in Table 1 . As displayed, a rate $8 / 11$ code with $k_{1}^{\text {even }}=1$ and $k_{1}^{\text {odd }}=2$ constraints is found, and this code has the $k=7$ constraint. Sequences of even lengths satisfying the $\left(k_{1}^{\text {even }}=\right.$ $1, k_{1}^{\text {odd }}=2, k$ ) constraint can be freely concatenated without violating the $\left(k_{1}^{\text {even }}=1, k_{1}^{\text {odd }}=2\right)$ constraint. Odd lengths sequences, however, cannot be freely concatenated without violating the $\left(k_{1}^{\text {even }}=2, k_{1}^{\text {odd }}=1\right)$ constraint. To solve this problem, assuming that there is a modulo-11 counter synchronized to the data, the two transitions in arrow can end at times $1,2,4,6,8$, and 10 relative to counter. The even and odd positions in a codeword of 11 bits are given as ( e o e o e o e o e o e). For example, a sequence of 3 codewords will be

$$
\begin{aligned}
& \left(\begin{array}{llllllllll}
10 & 9 & 8 & 7 & 6 & 5 & 4 & 3 & 2 & 1
\end{array}\right), \quad\left(\begin{array}{lllllllllll}
10 & 9 & 8 & 7 & 6 & 5 & 4 & 3 & 2 & 1
\end{array}\right),\left(\begin{array}{lllllllllll}
10 & 9 & 8 & 7 & 6 & 5 & 4 & 3 & 2 & 1
\end{array}\right)
\end{aligned}
$$

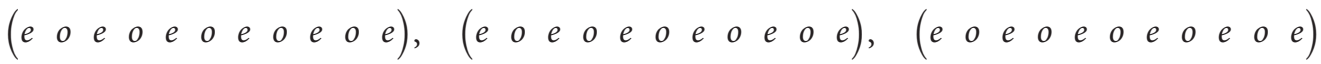

$$
\begin{aligned}
& \left(\begin{array}{lllllllllll}
1 & 2 & 1 & 2 & 1 & 2 & 1 & 2 & 1 & 2 & 1
\end{array}\right),\left(\begin{array}{lllllllllll}
1 & 2 & 1 & 2 & 1 & 2 & 1 & 2 & 1 & 2 & 1
\end{array}\right),\left(\begin{array}{lllllllllll}
1 & 2 & 1 & 2 & 1 & 2 & 1 & 2 & 1 & 2 & 1
\end{array}\right) \\
& \left(\begin{array}{lllllllllll}
x & e & o & e & o & e & o & e & o & e & o
\end{array}\right),\left(\begin{array}{lllllllllll}
x & e & o & e & o & e & o & e & o & e & o
\end{array}\right),\left(\begin{array}{lllllllllll}
x & e & o & e & o & e & o & e & o & e & o
\end{array}\right)
\end{aligned}
$$

where the 1st line expresses the positions of the code bits. The 2 nd line expresses the even/odd code bit positions. The 3rd line expresses the maximum number of consecutive " 1 " starting at the position. There is no two consecutive " 2 " in the 3rd line. It means that no dominant error event $\pm(1,-1)$ will occur. To obtain the coding gain of this encoder, a timevarying Viterbi detector is required. The trellis diagrams of the Viterbi detector for even and odd times are shown in Figure $7(\mathrm{c})$. The Viterbi detector for code bit stream positions will be the same as shifting the 2 nd line to right by 1 position. The result is shown in the 4 th line. The even or odd Viterbi detector properties must match the bit position shown in the 4 th line.

\section{Enumerative Encoding $\left(k_{1}^{\text {even }}=1, k_{1}^{\text {odd }}=2, k\right)$ TMTR Codes}

Let us lexicographically order the binary sequences of length $n$ by

$$
\begin{aligned}
\underline{X} & =\left(x_{n-1}, \ldots, x_{1}, x_{0}\right) \succ \underline{Y}=\left(y_{n-1}, \ldots, y_{1}, y_{0}\right) \\
& \Longleftrightarrow\left(x_{p}>y_{p}\right) \text { and }\left(x_{i}=y_{i}\right) \quad \forall p<i<n .
\end{aligned}
$$

An enumerating encoder maps a set of consecutive integers onto a lexicographically ordered set of sequences. In order to describe the enumerating encoder/decoder, some notations will be defined as follows.

(D.1) $A_{n}$ is the lexicographically ordered set of $\left(k_{1}^{\text {even }}=\right.$ $\left.1, k_{1}^{\text {odd }}=2, k ; r_{1}, r_{0}, l_{1}, l_{0}\right)=(1,2, k ; 1, k-1,1, \infty)$ sequences of length $n$.

(D.2) $R(\underline{\mathbf{X}})$ is the number of sequences $\underline{Y} \in A_{n}$ such that $\underline{X} \succ \underline{Y}$.
(D.3) $R(\underline{\mathbf{0}})=0$, where $\underline{0}$ is the all zero sequence.

(D.4) $\operatorname{res}(\underline{\mathbf{X}})$ is the sequence obtained by modifying the first nonzero bit of $\underline{X}$ to zero.

(D.5) $\underline{U}^{i}$ is the minimum sequence among sequences in $A_{n}$ and having the first symbol one at position $i$.

(D.6) $\underline{M}^{i}$ is the maximum sequence among sequences in $A_{n}$ and having the first symbol one at position $i$.

(D.7) $w_{i}=R\left(\underline{U}^{i}\right)-R\left(\operatorname{res}\left(\underline{U}^{i}\right)\right)$, then we have

$$
R(\underline{\mathbf{X}})=\sum_{i=0}^{n-1} x_{i} w_{i} .
$$

(D.8) We have $t_{i}=R\left(\underline{M}^{i}\right)$.

By definitions (D.5) and (D.6), it is easy to see that

$$
R\left(\underline{U}^{i}\right)=t_{i-1}+1 \text {. }
$$

The $w_{i}$ 's and $t_{i}$ 's can be obtained by the following recursive relation with initial values $w_{0}=1, t_{0}=1$ :

$$
\begin{aligned}
w_{i} & =t_{i-1}+1-R\left(\operatorname{res}\left(\underline{\mathbf{U}}^{i}\right)\right), \\
t_{i} & =R\left(\underline{\mathbf{M}}^{i}\right) .
\end{aligned}
$$

For illustration, consider $\left(k_{1}^{\text {even }}=1, k_{1}^{\text {odd }}=2, k ; r_{1}\right.$, $\left.r_{0}, l_{1}, l_{0}\right)=(1,2,4 ; 1,3,1, \infty)$ sequences with length 4 ; one has

$$
\begin{array}{clllll}
i & & 3 & 2 & 1 & 0 \\
w_{i} & \longrightarrow & 6 & 3 & 2 & 1 \\
t_{i} & \longrightarrow & 8 & 5 & 2 & 1 \\
R\left(\operatorname{res}\left(\underline{\mathbf{U}}^{i}\right)\right) & \longrightarrow & 0 & 0 & 0 & 0
\end{array}
$$


TABle 1: Practical code rates of $\left(k_{1}^{\text {even }}=1, k_{1}^{\text {odd }}=2, k\right)$ codes.

\begin{tabular}{lcc}
\hline Block length & Code rate & $\left(k_{1}^{\text {even }}=1, k_{1}^{\text {odd }}=2, k\right)$ \\
\hline 4 & $4 / 5$ & $(1,2,4)$ \\
5 & $3 / 5$ & $(1,2,4)$ \\
6 & $4 / 6$ & $(1,2,5)$ \\
7 & $5 / 7$ & $(1,2,6)$ \\
8 & $6 / 8$ & $(1,2,6)$ \\
9 & $7 / 9$ & $(1,2,7)$ \\
10 & $7 / 10$ & $(1,2,6)$ \\
11 & $8 / 11$ & $(1,2,7)$ \\
12 & $9 / 12$ & $(1,2,7)$ \\
20 & $14 / 20$ & $(1,2,6)$ \\
22 & $16 / 22$ & $(1,2,7)$ \\
24 & $18 / 24$ & $(1,2,7)$ \\
\hline
\end{tabular}

where

$$
\begin{array}{ll}
\underline{M}^{0}=(0001), & \underline{U}^{0}=(0001), \\
\underline{M}^{1}=(0010), & \underline{U}^{1}=(0010), \\
\underline{M}^{2}=(0110), & \underline{U}^{2}=(0100), \\
\underline{M}^{3}=(1010), & \underline{U}^{3}=(1000) .
\end{array}
$$

Similarly, when using a codeword of length 11 and code rate of $8 / 11$, the enumerating encoding method is as follows:

$$
\begin{aligned}
& \begin{array}{llllllllllll}
i & 10 & 9 & 8 & 7 & 6 & 5 & 4 & 3 & 2 & 1 & 0,
\end{array}
\end{aligned}
$$

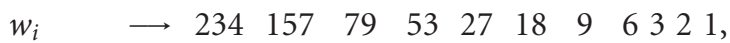

$$
\begin{aligned}
& t_{i} \quad \longrightarrow \quad 470236158 \quad 7953 \quad 26 \quad 17 \quad 85221 \text {, }
\end{aligned}
$$

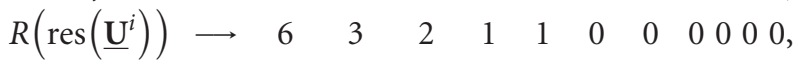

where

$$
\begin{aligned}
& \underline{M}^{0}=(00000001010), \quad \underline{U}^{0}=(00000001000), \\
& \underline{M}^{1}=(00000011010), \quad \underline{U}^{1}=(00000010000), \\
& \underline{M}^{2}=(00000101010), \quad \underline{U}^{2}=(00000100000), \\
& \underline{M}^{3}=(00001101010), \quad \underline{U}^{3}=(00001000000), \\
& \underline{M}^{4}=(00010101010), \quad \underline{U}^{4}=(00010000001), \\
& \underline{M}^{5}=(00110101010), \quad \underline{U}^{5}=(00100000001), \\
& \underline{M}^{6}=(01010101010), \quad \underline{U}^{6}=(01000000010), \\
& \underline{M}^{7}=(10110101010), \quad \underline{U}^{7}=(10000000100) .
\end{aligned}
$$

\begin{tabular}{|c|c|}
\hline $\begin{array}{l}\beta=\beta+t^{n-k-1}+1 \\
i=n-1 \\
\text { while } i>0 \\
\quad \text { if } \beta>t^{(i-1)} \\
\qquad x_{i}=1 \\
\quad \beta=\beta-w_{i} \\
\text { end } \\
\text { end } \\
x_{0}=\beta\end{array}$ & 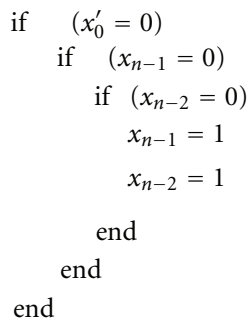 \\
\hline
\end{tabular}

Let $B_{n}$ be a subset of $A_{n}$ consisting of sequences with no more than $k=7$ leading zeros. Then the number of elements of $B_{11}$ is given as

$$
\begin{aligned}
\left|B_{11}\right| & =R([10110101010])-R([00000000110]) \\
& =w_{10}+w_{8}+w_{7}+w_{5}+w_{3}+w_{1}-w_{2}-w_{1} \\
& =387 .
\end{aligned}
$$

Figure 3: Enumerative encoding for $\left(k_{1}^{\text {even }}, k_{1}^{\text {odd }}, k\right)$ codes.

TABLE 2: Codebook of rate $3 / 4\left(k_{1}^{\text {even }}=1, k_{1}^{\text {odd }}=2, k=4\right)$ code.

\begin{tabular}{lccc}
\hline Data & Codeword & Data & Codeword \\
\hline$b_{1} \cdots b_{3}$ & $c_{1} \cdots c_{4}$ & $b_{1} \cdots b_{3}$ & $c_{1} \cdots c_{4}$ \\
000 & 0001 or 1101 & 100 & 0110 \\
001 & 0010 & 101 & 1000 \\
010 & 0100 & 110 & 1001 \\
011 & 0101 & 111 & 1010 \\
\hline
\end{tabular}

We can then encode an integer $\beta$ to a sequence $\underline{\mathbf{X}}$ using the enumerative algorithm given in Figure 3 (assuming the previous block is $\underline{\mathbf{X}}^{\prime}$ ). The decoding is simply done by

$$
\hat{\beta}=\sum_{i=0}^{n-1} x_{i} w_{i}-t^{n-k-1}-1
$$

where $t^{n-k-1}+1$ is the value of first codeword, $t_{i}=0$ and $t_{i}=-1$ for $i<0$.

For illustration, the mapping relationship between data and codewords of a rate $3 / 4$ code satisfying $\left(k_{1}^{\text {even }}=1, k_{1}^{\text {odd }}=\right.$ $2, k=4)$ constraint is given in Table 2 . The data to codeword mapping for a rate $8 / 11$ code satisfying $\left(k_{1}^{\text {even }}=1, k_{1}^{\text {odd }}=\right.$ $2, k=7)$ constraint is listed in Table 3.

\section{Power Spectral Density}

In DVD systems the power spectral density at low frequency, referred to as the low-frequency content, of the encoded data sequences should normally be maintained as low as possible to alleviate interference with pilot and focus servo signals. For example, in addition to satisfying the $(2,10)$ constraint, the 8/16 EFMPlus code employed in the DVD system is also designed to achieve very low low-frequency content to reduce the interference between the written signal and the servo signal. Efficient low-frequency component suppression is a crucial criterion for the $8 / 11\left(0, k_{1}^{\text {even }}=1, k_{1}^{\text {odd }}=2, k=7\right)$ TMTR code rate. The low-frequency content is based upon the running digital sum (RDS) $Z$ given by

$$
Z_{i}=\sum_{j=-\infty}^{i} X_{j}=Z_{i-1}+X_{i}
$$


TABLE 3: Data to codeword mapping for the rate $8 / 11\left(k_{1}^{\text {even }}=1, k_{1}^{\text {odd }}=2, k=7\right)$ code.

\begin{tabular}{|c|c|c|c|c|c|c|c|c|c|c|c|}
\hline & Data & Codeword & & Data & Codeword & & Data & Codeword & & Data & $\begin{array}{l}\text { Codeword } \\
c_{1} c_{2} \cdots c_{11}\end{array}$ \\
\hline & & & & & & & $b_{1} \cdots b_{8}$ & & & & \\
\hline \multirow[t]{2}{*}{0} & 00000000 & 00000001000 & 42 & 00101010 & 00001100100 & 94 & 01011110 & 00100100100 & 178 & 10110010 & 01001000000 \\
\hline & & or 11000001000 & & & or 1100110 & 95 & & 100100101 & 179 & 0110011 & 1001000001 \\
\hline \multirow[t]{2}{*}{1} & 00000001 & 00000001001 & 43 & 00101011 & 00001100101 & 96 & 100000 & 0100100110 & 180 & 0110100 & 1001000010 \\
\hline & & or 11000001001 & & & or 110 & 97 & 100001 & 1000 & 181 & 0110101 & 1001000100 \\
\hline \multirow[t]{2}{*}{2} & 00000010 & 000000010 & 44 & 00101100 & & 98 & 100010 & & 182 & 0110110 & 1001000101 \\
\hline & & or 11000001010 & & & or 11 & 99 & & & 183 & 110111 & 1001000110 \\
\hline \multirow[t]{2}{*}{3} & 00000011 & 00000010000 & 45 & 00101101 & & 100 & & & 184 & 111000 & 1001001000 \\
\hline & & or 11000010000 & & & or 1 & & & & 185 & 0111001 & 1001 \\
\hline \multirow[t]{2}{*}{4} & 00000100 & & 46 & 00101110 & & & & & 186 & 10 & \\
\hline & & or 1100 & & & or 1 & & & & 187 & & \\
\hline \multirow[t]{2}{*}{5} & 00000101 & & 47 & 00101111 & & & & & 188 & & 0001 \\
\hline & & or 1100 & & & or 1 & & & & 189 & & \\
\hline \multirow[t]{2}{*}{6} & 00000110 & 00000 & 48 & 00110000 & & & & & 190 & & \\
\hline & & or 1100 & & & or 1 & & & & 91 & & \\
\hline \multirow[t]{2}{*}{7} & 00000111 & 00000 & 49 & 00110001 & & & & & 192 & & \\
\hline & & or 1100 & & & or 1 & & & & 93 & & \\
\hline \multirow[t]{2}{*}{8} & 00001000 & 00000 & 50 & 00110010 & & 11 & & & 194 & & \\
\hline & & or 1100 & & & or 1 & & & & 95 & & \\
\hline \multirow[t]{2}{*}{9} & 00001001 & 00000 & 51 & 00110011 & & 112 & & & 96 & & \\
\hline & & or 1100 & & & or 1 & & & & 97 & & \\
\hline \multirow[t]{2}{*}{10} & 00001010 & 00000 & 52 & 00110100 & & 114 & & & 98 & & \\
\hline & & or 1100 & & & or 1 & & & & 99 & & \\
\hline \multirow[t]{2}{*}{11} & 00001011 & 000000 & 53 & 00110101 & & 116 & & & 200 & & \\
\hline & & or 11000 & & & or 1 & 11 & & & 201 & & \\
\hline \multirow[t]{2}{*}{12} & 00001100 & 00000 & 54 & 00110110 & & 118 & & & 202 & & \\
\hline & & or 1100 & & & or 1 . & & & & 03 & & \\
\hline \multirow[t]{2}{*}{13} & 00001101 & 00000 & 55 & 00110111 & & 120 & & & 204 & & \\
\hline & & or 110 & & & or 1 . & & & & 05 & & \\
\hline \multirow[t]{2}{*}{14} & 00001110 & 00000 & 56 & 00111000 & & 122 & & & 206 & & 0010 \\
\hline & & & & & & & & & & & \\
\hline \multirow[t]{2}{*}{15} & 00001111 & & 57 & 00111001 & & & & & 208 & & 101 \\
\hline & & & & & or 1 & & & & & & \\
\hline 16 & 00010000 & & 58 & 00111010 & & & & & & & \\
\hline & & & & & & & & & & & \\
\hline 17 & 00010001 & 0000 & 59 & & & & & & & & \\
\hline & & & & & & & & & & & \\
\hline 18 & 000100 & & 60 & & & & & & & & \\
\hline & & & & & & & & & & & \\
\hline 19 & 00010011 & & 61 & & & & & & & & \\
\hline & & & & & & & & & & & \\
\hline 20 & 00010100 & 00000 & 62 & 00111110 & & 134 & 10000110 & & 218 & 10 & 01010010110 \\
\hline & & or 11 & & & & & & & & & \\
\hline 21 & 00010101 & 00001000000 & 63 & 00111111 & 00010011001 & 136 & 10001000 & 00110010001 & 220 & 1011100 & 1010011001 \\
\hline & & or 11001000000 & & & or 11010011001 & 137 & 10001001 & 00110010010 & 221 & 1011101 & 01010011010 \\
\hline 22 & 00010110 & 00001000001 & 64 & 01000000 & & 138 & 10001010 & 00110010100 & 222 & 11011110 & 01010100000 \\
\hline & & & & & & & & & & & \\
\hline 23 & 00010111 & & 65 & 01000001 & & & & & & & \\
\hline & & & & & & & & & & & \\
\hline 24 & 00011000 & & 66 & 01000010 & & & & & & & \\
\hline & & & & & & & & & & & \\
\hline 25 & 00011001 & & 67 & 01000011 & & & & & 228 & & \\
\hline & & or 11001000101 & & & or 11010100010 & 145 & & 00110100001 & 229 & 11100101 & 0101010100 \\
\hline
\end{tabular}


TABle 3: Continued.

\begin{tabular}{|c|c|c|c|c|c|c|c|c|c|c|c|}
\hline & $\begin{array}{c}\text { Data } \\
b_{1} \cdots b_{8}\end{array}$ & $\begin{array}{c}\text { Codeword } \\
c_{1} c_{2} \cdots c_{11} \\
\end{array}$ & & $\begin{array}{c}\text { Data } \\
b_{1} \cdots b_{8}\end{array}$ & $\begin{array}{c}\text { Codeword } \\
c_{1} c_{2} \cdots c_{11}\end{array}$ & & $\begin{array}{c}\text { Data } \\
b_{1} \cdots b_{8}\end{array}$ & $\begin{array}{c}\text { Codeword } \\
c_{1} c_{2} \cdots c_{11}\end{array}$ & & $\begin{array}{c}\text { Data } \\
b_{1} \cdots b_{8} \\
\end{array}$ & $\begin{array}{l}\text { Codeword } \\
c_{1} c_{2} \cdots c_{11}\end{array}$ \\
\hline \multirow[t]{2}{*}{26} & 00011010 & 00001000110 & 68 & 01000100 & 00010100100 & 146 & 10010010 & 00110100010 & 230 & 11100110 & 01010101010 \\
\hline & & or 11001000110 & & & or 11010100100 & 147 & 10010011 & 00110100100 & 231 & 11100111 & 10000000100 \\
\hline \multirow[t]{2}{*}{27} & 00011011 & 00001001000 & 69 & 01000101 & 00010100101 & 148 & 10010100 & 00110100101 & 232 & 11101000 & 10000000101 \\
\hline & & or 11001001000 & & & or 11010100101 & 149 & 10010101 & 00110100110 & 233 & 11101001 & 10000000110 \\
\hline \multirow[t]{2}{*}{28} & 00011100 & 00001001001 & 70 & 01000110 & 00010100110 & 150 & 10010110 & 00110101000 & 234 & 11101010 & 10000001000 \\
\hline & & or 11001001001 & & & or 11010100110 & 151 & 10010111 & 00110101001 & 235 & 11101011 & 10000001001 \\
\hline \multirow[t]{2}{*}{29} & 00011101 & 00001001010 & 71 & 01000111 & 00010101000 & 152 & 10011000 & 00110101010 & 236 & 11101100 & 10000001010 \\
\hline & & or 11001001010 & & & or 11010101000 & 153 & 10011001 & 01000000010 & 237 & 11101101 & 10000010000 \\
\hline \multirow[t]{2}{*}{30} & 00011110 & 00001010000 & 72 & 01001000 & 00010101001 & 154 & 10011010 & 01000000100 & 238 & 11101110 & 10000010001 \\
\hline & & or 11001010000 & & & or 11010101001 & 155 & 10011011 & 01000000101 & 239 & 11101111 & 10000010010 \\
\hline \multirow[t]{2}{*}{31} & 00011111 & 00001010001 & 73 & 01001001 & 00010101010 & 156 & 10011100 & 01000000110 & 240 & 11110000 & 10000010100 \\
\hline & & or 11001010001 & & & or 11010101010 & 157 & 10011101 & 01000001000 & 241 & 11110001 & 10000010101 \\
\hline \multirow[t]{2}{*}{32} & 00100000 & 00001010010 & 74 & 01001010 & 00100000001 & 158 & 10011110 & 01000001001 & 242 & 11110010 & 10000010110 \\
\hline & & or 11001010010 & 75 & 01001011 & 00100000010 & 159 & 10011111 & 01000001010 & 243 & 11110011 & 10000011000 \\
\hline \multirow[t]{2}{*}{33} & 00100001 & 00001010100 & 76 & 01001100 & 00100000100 & 160 & 10100000 & 01000010000 & 244 & 11110100 & 10000011001 \\
\hline & & or 11001010100 & 77 & 01001101 & 00100000101 & 161 & 10100001 & 01000010001 & 245 & 11110101 & 10000011010 \\
\hline \multirow[t]{2}{*}{34} & 00100010 & 00001010101 & 78 & 01001110 & & & & & & & 10000100000 \\
\hline & & or 11001010101 & 79 & 01001111 & & 163 & & 01000010100 & 247 & 11110111 & 10000100001 \\
\hline \multirow[t]{2}{*}{35} & 00100011 & 00001010110 & 80 & 01010000 & 00100001001 & 164 & 10100100 & 01000010101 & 248 & 11111000 & 10000100010 \\
\hline & & or 11001010110 & 81 & 01010001 & 00100001010 & 165 & 10100101 & 01000010110 & 249 & 11111001 & 10000100100 \\
\hline \multirow[t]{2}{*}{36} & 00100100 & 00001011000 & 82 & 01010010 & 00100010000 & 166 & 10100110 & 01000011000 & 250 & 11111010 & 10000100101 \\
\hline & & or 11001011000 & 83 & 01010011 & 00100010001 & 167 & 10100111 & 01000011001 & 251 & 11111011 & 10000100110 \\
\hline \multirow[t]{2}{*}{37} & 00100101 & 00001011001 & 84 & 01010100 & 00100010010 & 168 & 10101000 & 01000011010 & 252 & 11111100 & 10000101000 \\
\hline & & or 11001011001 & 85 & 01010101 & 00100010100 & 169 & 10101001 & 01000100000 & 253 & 11111101 & 10000101001 \\
\hline \multirow[t]{2}{*}{38} & 00100110 & 00001011010 & 86 & 01010110 & 00100010101 & 170 & 10101010 & 01000100001 & 254 & 11111110 & 10000101010 \\
\hline & & or 11001011010 & 87 & 01010111 & 00100010110 & 171 & 10101011 & 01000100010 & 255 & 11111111 & 10001000000 \\
\hline \multirow[t]{2}{*}{39} & 00100111 & 00001100000 & 88 & 01011000 & 00100011000 & 172 & 10101100 & 01000100100 & & & \\
\hline & & or 11001100000 & 89 & 01011001 & 00100011001 & 173 & 10101101 & 01000100101 & & & \\
\hline \multirow[t]{2}{*}{40} & 00101000 & 00001100001 & 90 & 01011010 & 00100011010 & 174 & 10101110 & 01000100110 & & & \\
\hline & & or 11001100001 & 91 & 01011011 & 00100100000 & 175 & 10101111 & 01000101000 & & & \\
\hline \multirow[t]{2}{*}{41} & 00101001 & 00001100010 & 92 & 01011100 & 00100100001 & 176 & 10110000 & 01000101001 & & & \\
\hline & & or 11001100010 & 93 & 01011101 & 00100100010 & 177 & 10110001 & 01000101010 & & & \\
\hline
\end{tabular}

where $\left\{X_{i}\right\}=\left\{\ldots, X_{-1}, X_{0}, X_{1}, \ldots\right\} \in\{-1,+1\}$ represents the writing sequences. A lower RDS results in lower lowfrequency content. The power spectral density of a sequence can be expressed as

$$
H(w)=\lim _{M \rightarrow \infty} E\left[\frac{1}{2 M+1}\left|\sum_{m=-M}^{M} X_{m} e^{-j m w}\right|^{2}\right]
$$

For a bounded RDS, that is, $\left|Z_{i}\right|=\left|\sum_{j=-\infty}^{i} X_{j}\right| \leq C<\infty$, $C$ is a constant, and the $D C$ content is then given as

$$
H(0)=\lim _{M \rightarrow \infty} E\left[\frac{1}{2 M+1}\left|\sum_{m=-M}^{M} X_{m}\right|^{2}\right] \approx 0,
$$

that indicates a DC-free content. As shown in (11), the number of sequences with length 11 satisfying $\left(k_{1}^{\text {even }}=\right.$ $\left.1, k_{1}^{\text {odd }}=2, k, r_{1}, r_{0}, l_{1}, l_{0}\right)=(1,2,7 ; 1,6,1, \infty)$ is 387 , but it only requires 256 codewords in the rate 8/11 TMTR code. The surplus $387-256=131$ code sequences can be exploited for minimizing both $k$-constraint and lowfrequency content. The 131 surplus code sequences are then used to suppress the low-frequency content by reducing the running digital sum (RDS). There are two tables (a main table and a substitute table) used in the encoder, as shown in Figure 4. The main table consists of 256 codewords (i.e., sequences $\underline{\mathbf{X}}$ corresponding to $132 \leq \beta \leq 387$ in the enumerating algorithm), and the substitute table with 131 codewords (i.e., sequences $\underline{\mathbf{X}}$ corresponding to $1 \leq \beta \leq$ 131 ) is used to minimize the power spectral density at low frequencies. 


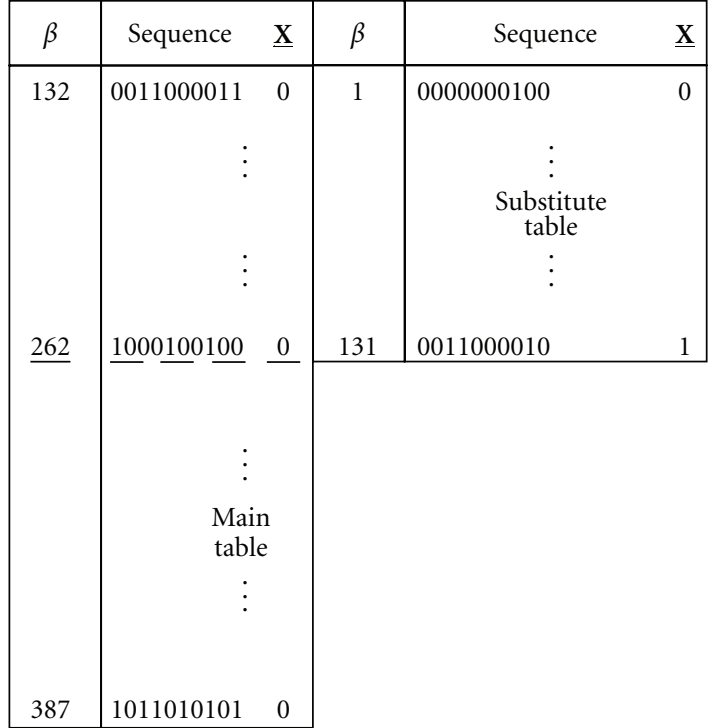

FIGURE 4: Codebook of rate $8 / 11\left(k_{1}^{\text {even }}=1, k_{1}^{\text {odd }}=2,7\right)$ TMTR code.

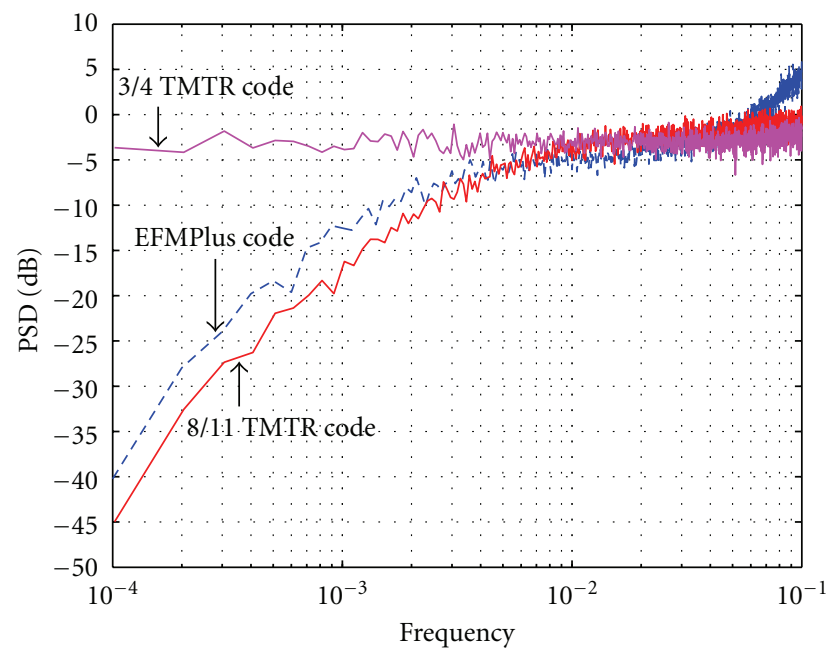

Figure 5: Power spectral density.

The PSD of both EFMPlus code and $\left(0, k_{1}^{\text {even }}=1, k_{1}^{\text {odd }}=\right.$ 2) TMTR code can be computed using the fast Fourier transform (FFT)

$$
H\left(k w_{s}\right) \cong \sum_{n=1}^{\infty} X_{n} W_{N}^{(k-1)(n-1)},
$$

where $W_{N}^{k}=e^{-j(2 k \pi / N)}, w_{s}=2 \pi / N$.

Figure 5 depicts the power spectral density (PSD) versus the normalized frequency for the 8/16 EFMPlus code, the rate $3 / 4\left(0, k_{1}^{\text {even }}=1, k_{1}^{\text {odd }}=2, k=4\right)$ TMTR code, and the $8 / 11\left(0, k_{1}^{\text {even }}=1, k_{1}^{\text {odd }}=2, k=7\right)$ TMTR code with method of enumerative, respectively. As shown, at frequency of $10^{-4}$, the PSDs for both EFMPlus and TMTR codes are $-40 \mathrm{~dB}$, $-4 \mathrm{~dB}$ and $-45 \mathrm{~dB}$, respectively. The result indicates that the $8 / 11\left(0, k_{1}^{\text {even }}=1, k_{1}^{\text {odd }}=2, k=7\right)$ TMTR code achieves

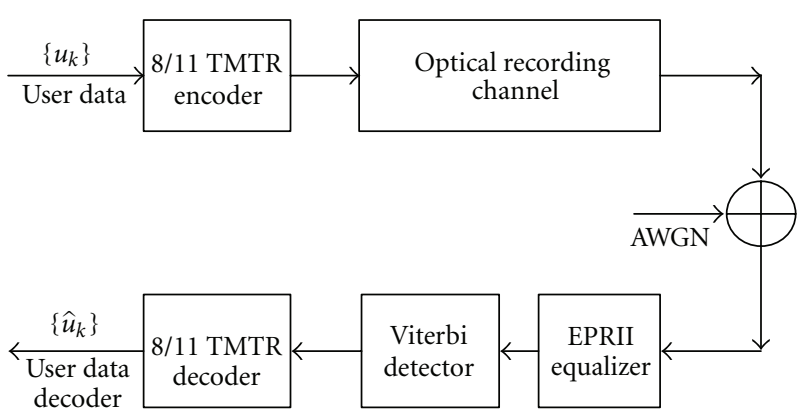

FIGURE 6: Optical recording system model.

a lower low-frequency content (with $5 \mathrm{~dB}$ lower) than the EFMPlus code. In Figure 5, we notice that the rate 3/4 $\left(0, k_{1}^{\text {even }}=1, k_{1}^{\text {odd }}=2, k=4\right)$ TMTR code has only $-4 \mathrm{~dB}$ at frequency of $10^{-4}$ and it is not suitable for optical recording systems, although it has a higher code rate compared to the $8 / 11\left(0, k_{1}^{\text {even }}=1, k_{1}^{\text {odd }}=2, k=7\right)$ TMTR code.

\section{Simulation Results}

The superiority of the rate $8 / 11\left(0, k_{1}^{\text {even }}=1, k_{1}^{\text {odd }}=2\right)$ TMTR code over the EFMPlus code is also demonstrated on error performance through a computer simulation on the EPRII optical channel of the form $P(D)=(1+D)^{3}$. Figure 6 depicts the optical recording system for simulation. An 8-state transition diagram, as depicted in Figure 7(a), can describe the EPRII optical channel. During simulation, a Gaussian optical recording channel was assumed with impulse response $m(t)$ given by

$$
m(t)=\frac{2}{\sqrt{\pi} S T} \exp \left\{-\left(\frac{2 t}{S T}\right)^{2}\right\},
$$

where $S$ represents the user information density. $T$ is the channel bit period assumed to be one $(T=1)$ for uncoded systems in simulation. The optical recording channel is then corrupted with the additive white Gaussian noise (AWGN), and at receiver an ideal EPRII equalizer is employed to equalize the optical channel in the form of $P(D)=(1+D)^{3}$.

The Viterbi detector is then employed on the 8-state trellis diagram of the EPRII optical channel, to recover binaryrecorded data from the equalized and sampled output. Note that for the EFMPlus-coded EPRII channel the 8-state trellis diagram can be reduced to a 6-state trellis diagram due to the $(2,10)$ constraint, as shown in Figure $7(b)$, while for the TMTR-coded EPRII channel the 8-state trellis diagram is still required with some branches deleted as depicted in Figure $7(\mathrm{c})$. Figure 8 demonstrates the bit error rate versus the signal to noise ratio (SNR) for TMTR-coded, EFMPluscoded and uncoded EPRII optical recording channels at user density $S=1.2$. The $\mathrm{SNR}$ in $\mathrm{dB}$ is defined in this paper as the ratio of the input complex waveform signal energy generated by a 127-bit pseudorandom binary sequence to the channel noise energy of the same duration. As shown both the TMTR-coded and EFMPlus-coded EPRII systems achieve better performance than the uncoded EPRII system. 


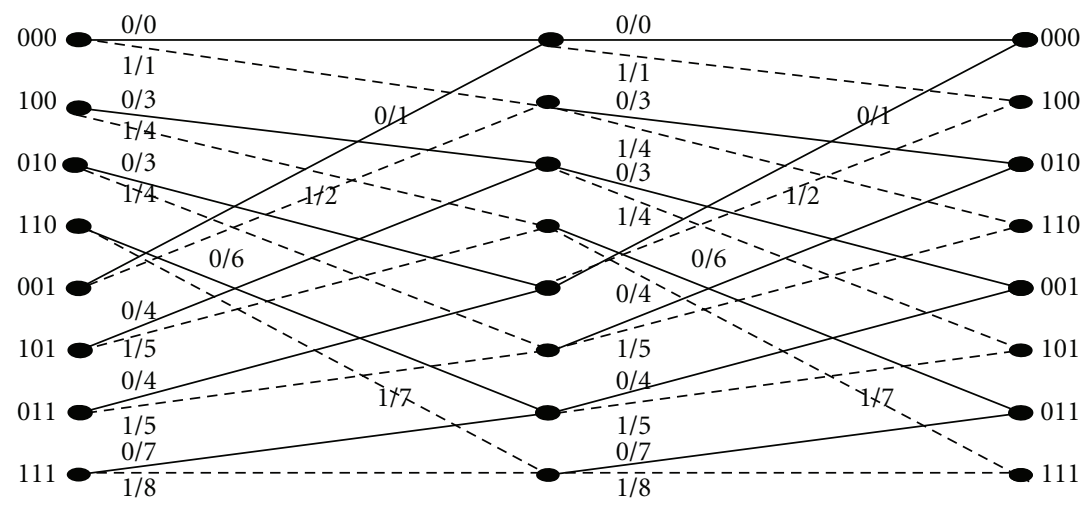

(a)

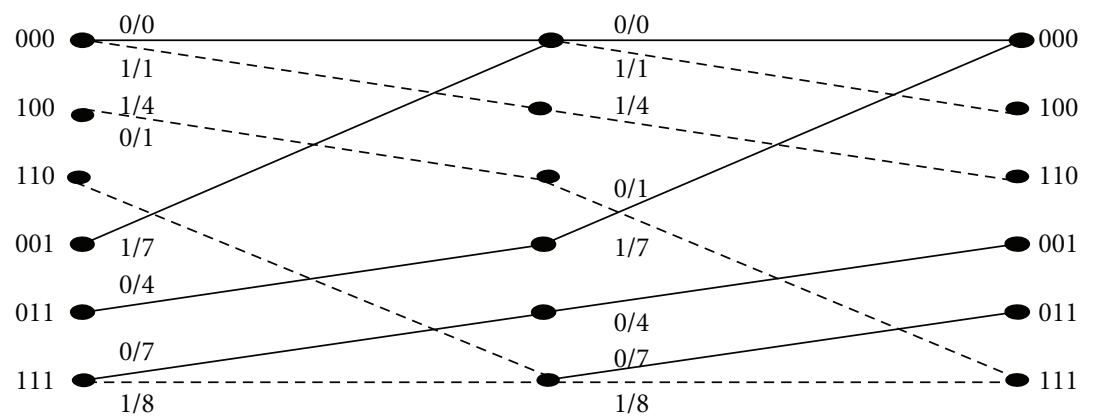

(b)

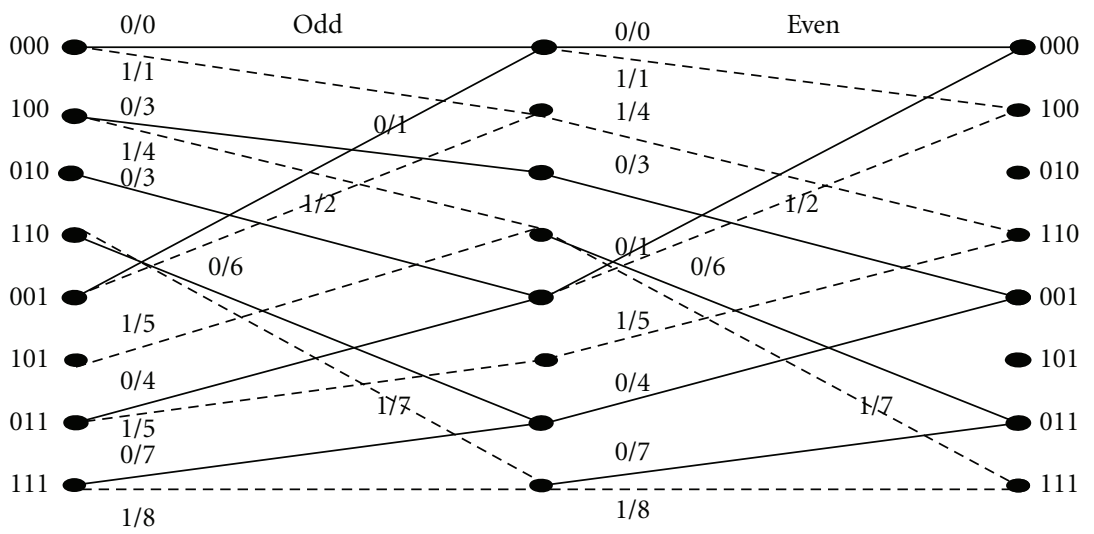

(c)

FIgURE 7: Trellis diagram. (a) EPRII channel. (b) EFMPlus-coded EPRII channel. (c) TMTR-coded EPRII channel.

This is because both coded EPRII systems have a coding gain of $3 \mathrm{~dB}$ when these modulation codes are considered in the 8-state trellis diagram of the EPRII system during detection. This leads to a coding gain of $3 \mathrm{~dB}$. As also can be seen in this figure, the TMTR-coded EPRII system improves the EFMPlus-coded EPRII system by approximately $1 \mathrm{~dB}$ in bit error rate. The coding loss of the EFMPlus-coded EPRII system is due to the bit rate loss. Figure 9 shows the signal to noise (SNR) required to achieve a bit rate error of $10^{-5}$, as a function of user density, at a rate of $8 / 11\left(0, k_{1}^{\text {even }}=1, k_{1}^{\text {odd }}=\right.$ 2) TMTR code and a rate of $8 / 16$ EFMPlus code, applied to an EPRII optical channel. Figure 9 shows how the TMTR code provides little coding gain at user densities below 1.2 but increases coding gain at higher densities. At a user density of $S=2.0$, the TMTR code on the EPRII optical channel provides nearly $2.7 \mathrm{~dB}$ of coding gain above the rate of $8 / 16$ EFMPlus code on the EPRII optical channel. Therefore, from the performance comparison made in Figure 9, it can be seen that even a greater improvement in coding gain could be achieved for TMTR-coded EPRII system at higher user densities.

\section{Conclusion}

In this paper, we present an enumeration method for constructing $\left(k_{1}^{\text {even }}=1, k_{1}^{\text {odd }}=2, k\right)$ codes, and the lookup table described in a previous work is not required for the encoder/decoder. Based on the construction, a rate 


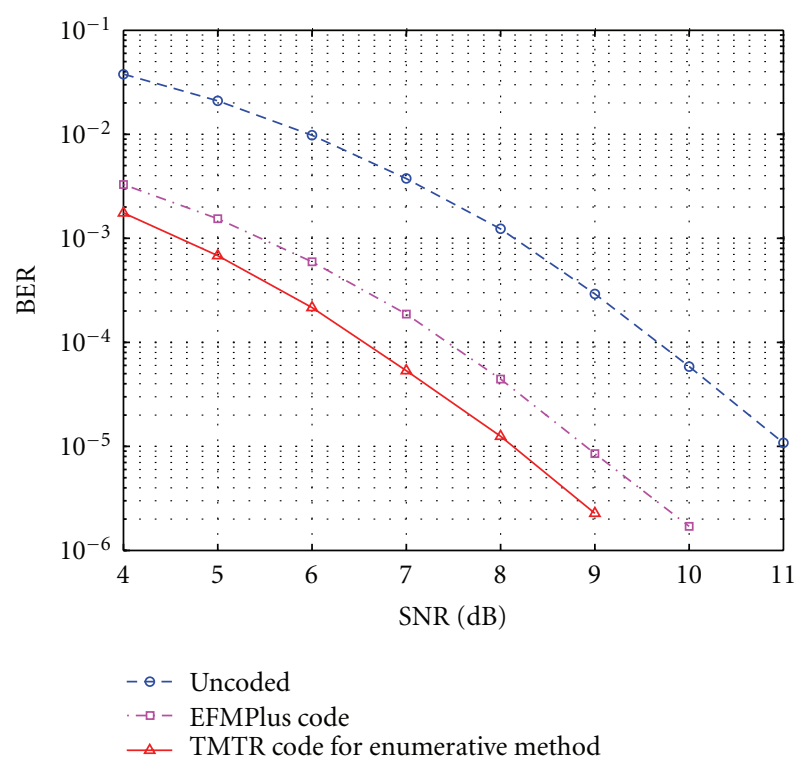

FIGURE 8: Error performance of TMTR-/EFMPlus-coded and uncoded EPRII systems.

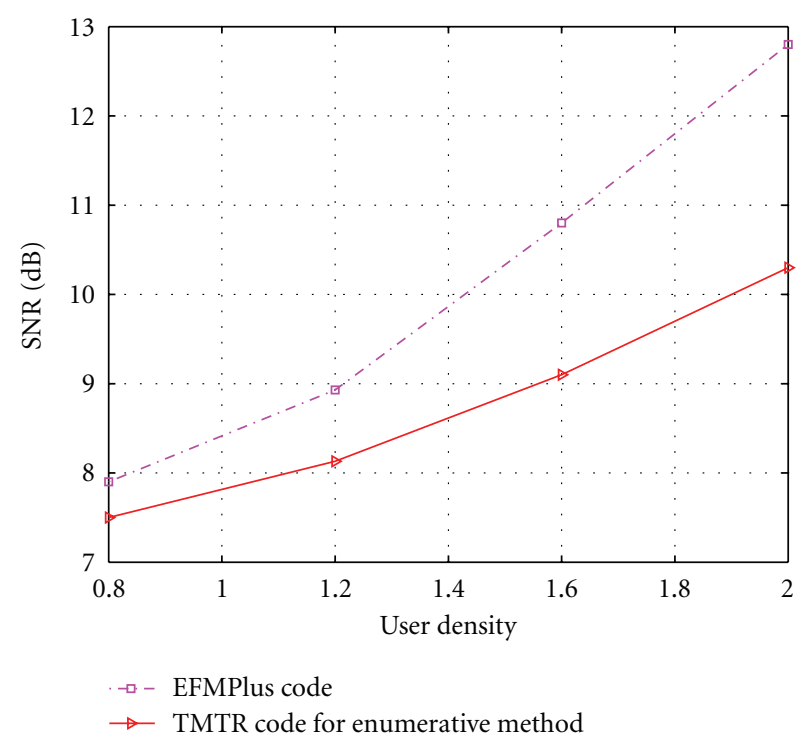

FIGURE 9: The SNR in $\mathrm{dB}$ required to achieve a $10^{-5}$ BER versus user density.

$8 / 11\left(k_{1}^{\text {even }}=1, k_{1}^{\text {odd }}=2,7\right)$ TMTR code is found. This code can achieve lower power spectral density at low frequencies compared to the EFMPlus code. In addition, computer simulations reveal that the rate 8/11 TMTR code outperforms the EFMPlus code in error performance when applied to partial response optical recording channels.

\section{References}

[1] K. A. S. Immink, "EFMPlus: the coding format of the multimedia compact disc," IEEE Transactions on Consumer Electronics, vol. 41, no. 3, pp. 491-497, 1995.
[2] J. Moon and B. Brickner, "Maximum transition run codes for data storage systems," IEEE Transactions on Magnetics, vol. 32, no. 5, pp. 3992-3994, 1996.

[3] B. E. Moision, P. H. Siegel, and E. Soljanin, "Distanceenhancing codes for digital recording," IEEE Transactions on Magnetics, vol. 34, no. 1, pp. 69-74, 1998.

[4] W. G. Bliss, "An 8/9 rate time-varying trellis code for high density magnetic recording," IEEE Transactions on Magnetics, vol. 33, no. 5, pp. 2746-2748, 1997.

[5] K. K. Fitzpatrick and C. S. Modlin, "Time-varying MTR codes for high density magnetic recording," in Proceedings of the IEEE Global Telecommunications Conference (GLOBECOM '97), pp. 1250-1253, Phoenix, Ariz, USA, November 1997.

[6] B. Nikolić, M. M.-T. Leung, and L. K.-C. Fu, "Rate 8/9 sliding block distance-enhancing code with stationary detector," IEEE Transactions on Magnetics, vol. 37, no. 3, pp. 1168-1174, 2001.

[7] R. D. Cideciyan and E. Eleftheriou, "Codes satisfying maximum transition run and parity-check constraints," in Proceedings of the IEEE International Conference on Communications, pp. 635-639, June 2004.

[8] I. Demirkan and Y. X. Lee, "The combined constraints for perpendicular recording channels," IEEE Transactions on Magnetics, vol. 42, no. 2, pp. 220-225, 2006.

[9] T. L. Poo and B. H. Marcus, "Time-varying maximum transition run constraints," IEEE Transactions on Information Theory, vol. 52, no. 10, pp. 4464-4480, 2006.

[10] H.-F. Tsai and Y. Lin, "Turbo decoding for a new DVD recording system," IEEE Transactions on Consumer Electronics, vol. 51, no. 3, pp. 864-871, 2005.

[11] R. D. Cideciyan, F. Dolivo, R. Hermann, W. Hirt, and W. Schott, "A PRML system for digital magnetic recording," IEEE Journal on Selected Areas in Communications, vol. 10, no. 1, pp. 38-56, 1992.

[12] R. Karabed and P. H. Siegel, "Matched spectral-null codes for partial-response channels," IEEE Transactions on Information Theory, vol. 37, no. 3, pp. 818-855, 1991.

[13] G. Vannucci and G. J. Foschini, "The minimum distance for digital magnetic recording partial responses," IEEE Transactions on Information Theory, vol. 37, no. 3, pp. 955-960, 1991. 